Ophthalmologe 2015 - 112:1017-1021

DOI 10.1007/s00347-015-0162-z

Online publiziert: 24. November 2015

(c) Die Autor(en) 2015. Dieser Artikel ist auf

Springerlink.com mit Open Access verfügbar

CrossMark

B. Schuler-Thurner ${ }^{9} \cdot$ K. -U. Bartz-Schmidt ${ }^{1} \cdot$ N. Bornfeld ${ }^{2} \cdot$ C. Cursiefen ${ }^{3}$.

B. Fuisting ${ }^{4} \cdot$ S. Grisanti ${ }^{5}$. L.M. Heindl ${ }^{3} \cdot$ L. Holbach ${ }^{6} \cdot$ M. Keserü $^{4}$.

H. Knorr ${ }^{6} \cdot$ K. Koch ${ }^{3} \cdot$ F. Kruse ${ }^{6} \cdot$ R. Meiller $\cdot$ C. Metz ${ }^{2}$ T. Meyer-ter-Vehn ${ }^{7} \cdot$ M. Much ${ }^{7}$. M. Reinsberg ${ }^{5} \cdot$ S. Schliep ${ }^{9} \cdot$ B. Seitz ${ }^{8} \cdot$ G. Schuler $^{9} \cdot$ D. Süsskind ${ }^{1} \cdot$ A. Viestenz ${ }^{8}$. L. Wagenfeld ${ }^{4} \cdot M$. Zeschnigk ${ }^{10}$

${ }^{1}$ Augenklinik, UK Tübingen, Tübingen, Deutschland

${ }^{2}$ Augenklinik, UK Essen, Essen, Deutschland

${ }^{3}$ Zentrum für Augenheilkunde, UK Köln, Köln, Deutschland

${ }^{4}$ Klinik und Poliklinik für Augenheilkunde, UK Hamburg-Eppendorf, Hamburg-Eppendorf, Deutschland

${ }^{5}$ Augenklinik, UK Lübeck, Lübeck, Deutschland

${ }^{6}$ Augenklinik, UK Erlangen, Erlangen, Deutschland

${ }^{7}$ Augenklinik, JMU Würzburg, Würzburg, Deutschland

${ }^{8}$ Klinik für Augenheilkunde, UK des Saarlandes, Homburg, Deutschland

${ }^{9}$ Experimentelle Immuntherapie, Hautklinik, Universitätsklinikum Erlangen, Erlangen, Deutschland

${ }^{10}$ Institut für Humangenetik, Ophthalmologische Onkologie und Genetik, UK Essen, Essen, Deutschland

\title{
Immuntherapie beim Aderhautmelanom: Vakzination gegen Krebs
}

\author{
Multizentrische adjuvante Phase-III- \\ Impfstudie mit Tumor-RNA-beladenen \\ dendritischen Zellen bei neu \\ diagnostizierten, großen Uveamelanomen
}

\section{Uveamelanom und Immunsystem}

Das maligne Melanom der Aderhaut ist der häufigste primäre intraokulare maligne Tumor des Erwachsenenalters [1-3]. Die Inzidenz liegt bei etwa 5:100.000 pro Jahr [1-3]. Die Inzidenz steigt mit zunehmendem Lebensalter [13]. Das Melanom der Aderhaut führt oft erst spät zu Symptomen. Die Prognose quoad vitam des Aderhaumelanoms ist schlecht, etwa $50 \%$ der Patienten entwickeln Fernmetastasen [1-3]. Im Gegensatz zum kutanen Melanom zeigt das uveale Melanom einen starken Tropismus zur Lebermetastasierung und metastasiert (bis auf Tumoren mit extraokularer Ausdehnung) ausschließlich hämatogen [4]. Dies scheint in der Lymphgefäß-

Autoren in alphabetischer Reihenfolge freiheit der normalen Aderhaut und der alymphatischen Sklerabarriere begründet zu sein [5-6]. Eine weitere Besonderheit des uvealen Melanoms ist die relative Sicherheit vor dem körpereigenen Immunsystem durch die Lage im „immunprivilegierten“ Auge. Anders als kutane Melanome ist der intraokulare Tumor dem Zugriff des Immunsystems teilweise entzogen. Deshalb versucht der hier vorgestellte neue Therapieansatz einer „personalisierten Impftherapie“ dem Immunsystem beim Angriff gegen den Tumor gezielt zu helfen.

Denn unabhängig von der Therapie des Primärtumors am Auge (Enukleation, Endoresektion, Blockexzision, Brachytherapie, Protonenbestrahlung etc.) ist die Prognose quoad vitam schlecht und bisher durch adjuvante Therapieansätze nicht zu beeinflussen [1-3]. Besonders Aderhautmelanome mit Monosomie 3 sind mit einer dramatisch schlechten Prognose vergesellschaftet [7], da es meist bald zum Auftreten von Lebermetastasen und in Folge innerhalb von Monaten zum Tode des Patienten kommt. Dieser Verlauf kann auch in Zeiten von Kinaseinhibitoren und Checkpoint-Blockade nur in Einzelfällen verzögert werden [1-3]. Somit gibt es zurzeit für Aderhautmelanompatienten sowohl in der adjuvanten als auch in der metastasierten Situation keine zufriedenstellende zugelassene Therapieoption [810]. Der Ansatz einer personalisierten „Tumorimpfung“ des Patienten gegen das geschützt im Augeninneren liegende Tumorgewebe stellt ein Erfolg versprechendes neues Therapiekonzept, speziell für Hochrisikopatienten mit Aderhautmelanom und Monosomie 3, dar. 


\begin{tabular}{|c|c|c|}
\hline Tumorentität & Unternehmen & $\begin{array}{l}\text { Studiennummer oder } \\
\text { zugelassenes Medikament }\end{array}$ \\
\hline Nierenzellkarzinom & Argos Therapeutics, USA & NCT01582672 \\
\hline \multirow[t]{2}{*}{ Prostatakarzinom } & Dendreon, USA & Provenge $^{\circledast}$ \\
\hline & Sotio, Tschechei & NCT02111577 \\
\hline \multirow[t]{2}{*}{ Glioblastom } & Northwest Biotherapeutics, USA & NCT00045968 \\
\hline & $\begin{array}{l}\text { Immunocellular Therapeutics, USA } \\
\text { Pharmacell, Niederlande }\end{array}$ & Geplant \\
\hline Melanom der Haut & Caladrius Biosciences, USA & NCT01875653 \\
\hline Kolonkarzinom & Dandrit Biotech, Dänemark & Geplant \\
\hline Uveamelanom & Universitätsklinikum Erlangen, Hautklinik & NCT01983748 \\
\hline
\end{tabular}

Tab. 2 Teilnehmende Universitätskliniken

\begin{tabular}{|llll}
\hline Erlangen & $\begin{array}{l}\text { Prof. Friedrich Kruse, Dr. Harald } \\
\text { Knorr, Dr. Ralph Meiller }\end{array}$ & $\begin{array}{l}09131 / 8544141 \\
\text { oder 8534478 }\end{array}$ & $\begin{array}{l}\text { Barbara.stoll@uk-erlangen. } \\
\text { de }\end{array}$ \\
\hline Essen & $\begin{array}{l}\text { Prof. Norbert Bornfeld, } \\
\text { Dr. Claudia Metz }\end{array}$ & $\begin{array}{l}0201 / 72383471 \\
\text { oder } 83474\end{array}$ & claudia.metz@uk-essen.de \\
\hline $\begin{array}{l}\text { Hamburg } \\
\text { Eppendorf }\end{array}$ & $\begin{array}{l}\text { PD Lars Wagenfeld, Dr. Bettina } \\
\text { Fuisting, Dr. Matthias Keserü }\end{array}$ & $040 / 741052350$ & augenklinik@uke.de \\
\hline $\begin{array}{l}\text { Homburg/ } \\
\text { Saar }\end{array}$ & $\begin{array}{l}\text { PD Dr. Arne Viestenz, } \\
\text { Prof. Berthold Seitz }\end{array}$ & $06841 / 1622302$ & arne.viestenz@uks.eu \\
\hline Köln & $\begin{array}{l}\text { PD Dr. Ludwig M. Heindl, } 1622387 \\
\text { Prof. Claus Cursiefen }\end{array}$ & $0221 / 4784308$ & $\begin{array}{l}\text { augenklinik-studien@uk- } \\
\text { koeln.de }\end{array}$ \\
\hline Lübeck & $\begin{array}{l}\text { Prof. Salvatore Grisanti, } \\
\text { Dr. Schulz-Wagenbarth }\end{array}$ & $0451 / 5002217$ & salvatore.grisanti@uk-sh.de \\
\hline Tübingen & $\begin{array}{l}\text { PD Dr. Daniela Süsskind, } \\
\text { Prof. Dr. Karl-Ulrich Bartz-Schmidt }\end{array}$ & $07071 / 2983730$ & $\begin{array}{l}\text { daniela.suesskind@med.uni- } \\
\text { tuebingen.de }\end{array}$ \\
\hline Würzburg & $\begin{array}{l}\text { Dr. Tobias Meyer-ter-Vehn, } \\
\text { Dr. Martin Much }\end{array}$ & $\begin{array}{l}0931 / 20120458 \\
\text { oder 20614 }\end{array}$ & Hermann_C1@ukw.de \\
& & &
\end{tabular}

\section{Vakzination mit dendritischen Zellen}

Die Vakzination mit dendritischen Zellen ist eine Methode der Krebsimmuntherapie, die bereits bei mehreren Tumorentitäten die Phase III erreicht hat (• Tab. 1), darunter Nierenzellkarzinom, Prostatatakarzinom, Glioblastom, kutanes Melanom, Kolonkarzinom und Uveamelanom. Ein Präparat (Provenge ${ }^{\varpi}$ ) für die Behandlung von metastasiertem Prostatakarzinom ist bereits in den USA und Europa zugelassen. Die aktuelle Phase-III-Studie für das Uveamelanom ist die einzige Studie, welche nicht von Firmen, sondern ausschließlich von Universitätskliniken mit Unterstützung der Deutschen Krebshilfe betrieben wird.

Dendritische Zellen sind immunregulatorische Zellen, die die Funktion des Immunsystems modulieren können, indem sie entweder Immunität oder Toleranz gegenüber bestimmten Anti- genen induzieren. Dafür werden dendritische Zellen ex vivo in geeigneter Weise angezogen, dann 1) durch sog. Reifung in den "stimulatory mode" versetzt und 2) mit spezifischen Antigenen (z. B. Tumorantigenen) beladen. Die so behandelten Zellen werden dem Patienten verabreicht, wo sie durch Aktivierung von zytotoxischen CD8und CD4-Helfer-T-Zellen, welche gegen die geimpften Tumorantigene gerichtet sind, eine Immunantwort gegen die geimpften Tumorantigene induzieren. Dies führt zur Elimination der Tumorzellen durch das eigene Immunsystem. Die Immuninduktion ist antigenspezifisch, das Immunsystem wird ausschließlich gegen die geimpften Antigene in Gang gesetzt. Im Rahmen der Studie werden die dendritischen Zellen des Patienten mit RNA beladen, die aus dem Tumor des Patienten extrahiert und mittels "polymerase chain reaction“ (PCR) amplifiziert wurde. Diese auto- loge Tumor-RNA repräsentiert die im Tumor aktiven Gene und bildet somit auch ab, was auf den Tumorzellen als Antigen präsentiert wird. Kommt es durch die Impfung zur Induktion tumorantigenspezifischer Zellen, können im Körper verbliebene Tumorzellen erkannt und durch das aktivierte Immunsystem eliminiert werden.

Wenn sich die Erfolge aus der Behandlung von kutanem Melanom, Prostatakarzinom, Nierenzellkarzinom, Glioblastom und vielen anderen Tumorentitäten auf das Uveamelanom übertragen lassen, ist eine Verlängerung des Überlebens durch Verzögerung der Metastasierung zu erwarten, im besten Fall kann die Behandlung eine Progression durch die Induktion zytotoxischer T-Zellen gegen patienteneigene Tumorantigene vollständig unterbinden.

\section{Phase-III-Vakzinationsstudie beim Uveamelanom}

Die Universitätsaugenkliniken Erlangen, Essen, Hamburg-Eppendorf, Homburg/ Saar, Köln, Lübeck, Tübingen und Würzburg führen eine multizentrische klinische Phase-IIII-Studie durch, die Patienten mit neu diagnostiziertem und großem Uveamelanom (T2-T4, AJCC TNM grading 2009) eine personalisierte Immuntherapie anbietet. Das Ziel der Studie ist es, das Auftreten von Metastasen durch Aktivierung zytotoxischer T-Zellen zu verhindern.

Untersucht wird ein sog. ATMP („advanced therapy medicinal product“), eine autologe personalisierte Vakzine, die aus dendritischen Zellen des Patienten besteht, welche zuvor mit Tumor-RNA beladen wurden, die aus dem Uveamelanom des Patienten stammt. Ziel ist es, die dendritischen Zellen so mit einem Abbild des unikalen Tumorantigenrepertoires zu beladen, um damit - zurück im Patienten - Killerzellen zu aktivieren, die spezifisch gegen die individuellen Tumorantigene des Patienten gerichtet sind.

Dazu wird im Rahmen der Operation des Primärtumors (vorzugsweise Enukleation) ein mindestens „erbsengroßes“ Tumorgewebestück entnommen, aus dem dann in einem Reinraumlabor RNA isoliert wird. Diese wird mittels 
Ophthalmologe 2015 · 112:1017-1021 DOI 10.1007/s00347-015-0162-z

(c) Die Autor(en) 2015. Dieser Artikel ist auf Springerlink.com mit Open Access verfügbar

B. Schuler-Thurner · K.-U. Bartz-Schmidt · N. Bornfeld · C. Cursiefen · B. Fuisting · S. Grisanti · L.M. Heindl · L. Holbach ·

M. Keserü · H. Knorr · K. Koch · F. Kruse · R. Meiller · C. Metz · T. Meyer-ter-Vehn · M. Much · M. Reinsberg · S. Schliep · B. Seitz ·

G. Schuler $\cdot$ D. Süsskind $\cdot$ A. Viestenz $\cdot$ L. Wagenfeld $\cdot$ M. Zeschnigk

Immuntherapie beim Aderhautmelanom: Vakzination gegen Krebs.

Multizentrische adjuvante Phase-III-Impfstudie mit Tumor-RNA-beladenen dendritischen Zellen bei neu diagnostizierten, großen Uveamelanomen

\section{Zusammenfassung}

Uveamelanome sind die häufigsten malignen Augentumoren. Mit modernen molekularbiologischen Methoden (Chromosom-3Typisierung, Genexpressionsanalyse) können weniger aggressive von hoch aggressiven Verlaufsformen (Monosomie 3, Klasse II) unterschieden werden. Diese molekularbiologische Stratifizierung hat bisher ausschließlich eine Bedeutung für die Risikoeinschätzung, da es keine wirksame Therapie gibt, die eine Metastasierung verhindern oder verzögern kann. Eine randomisierte Studie mit einer Krebsvakzine bestehend aus patienteneigenen dendritischen Zellen und patienteneigener Uveamelanom-RNA soll zeigen, ob die Vakzine wie erwartet bei Patienten mit schlechter Prognose eine Metastasierung verzögern oder verhindern kann. Ein Einschluss in die Studie und damit ein potenzieller Vorteil für den Patienten ist nur möglich, sofern der Patient noch vor Operation oder Bestrahlung an einem der teilnehmenden Zentren vorgestellt wird, da für die Vakzinenherstellung Tumormaterial unter standardisierten Bedingungen gewonnen werden muss.

\section{Schlüsselwörter}

Uveamelanom · Dendritische Zellen . Krebsimpfung $\cdot$ Krebsimmuntherapie . Monosomie 3

\section{Immunotherapy of uveal melanoma: vaccination against cancer. Multicenter adjuvant phase 3 vaccination study using dendritic cells laden with tumor RNA for large newly diagnosed uveal melanoma}

\section{Abstract}

Uveal melanomas are the most common malignant tumors of the eye. With modern molecular biological diagnostic methods, such as chromosome 3 typing and gene expression analysis, these tumors can be categorized into highly aggressive (monosomy 3 , class II) and less aggressive forms. This molecular biological stratification is primarily important for determining the risk of these tumors as no therapy is currently available that is able to prevent or delay metastases. A ran- domized study of patients with a poor prognosis (monosomy 3 ) is currently being carried out in order to determine whether a cancer vaccine prepared from autologous (patient's own) dendritic cells and uveal melanoma RNA can prevent or delay progression and further metastases of this extremely aggressive form of cancer. Inclusion in the uveal melanoma study, which hopes to provide a potential therapeutic option for patients, is only possible if patients are referred to an in- stitution that is able to manufacture and provide this vaccination before the patient is operated on or treated with radiation. Untreated tumor material is necessary for producing the vaccine on an individualized patient basis.

\section{Keywords}

Uveal melanoma · Dendritic cells .

Cancer vaccination $\cdot$ Immunotherapy, cancer . Monosomy 3
Elektroporation in die vom jeweiligen Patienten gezüchteten dendritischen Zellen eingebracht. Die fertige Vakzine wird eingefroren an die zuweisenden Zentren versendet und vor Ort in den Studienzentren dem Patienten infundiert ([11, 12]; • Abb. 1).

Um an der Studie teilnehmen zu können, müssen Patienten mit Verdacht auf Uveamelanom noch vor der Therapie des Primärtumors (Operation oder Bestrahlung) an einem der teilnehmenden Zentren (• Tab. 2) vorgestellt werden. ${ }^{1}$ Nach erfolgter Primärtherapie außer-

1 Kontaktaufnahme bitte entweder über die in - Tab. 2 angegebenen teilnehmenden Augenkliniken oder über experimentelle-immuntherapie@uk-erlangen.de, Tel. 091318545833. halb eines Studienzentrums ist eine Inklusion in die Studie nicht mehr möglich, da das Tumormaterial unter standardisierten und kontrollierten Bedingungen entnommen und zur zentralisierten Produktionsstätte der Vakzine (Experimentelle Immuntherapie, Hautklinik, Universitätsklinikum Erlangen) transportiert werden muss.

Für die Gewinnung der autologen Tumor-RNA muss ein Teil des Uveamelanoms in einer Lösung, welche den Abbau von RNA durch Blockade von RNAsen verhindert, in ein Reinraumlabor am Universitätsklinikum Erlangen (Experimentelle Immuntherapie, Hautklinik) transportiert werden, wo die RNA unter GMP („good manufacturing practice“) -Bedingungen extrahiert und amplifiziert wird (• Abb. 1). Für die Anzucht der dendritischen Zellen müssen durch eine Leukapherese Vorläuferzellen (Monozyten) des Patienten gewonnen werden, die sich im Verlauf einer Woche unter Zugabe von Zytokinen (GM-CSF, Interleukin 4) sowie Reifungsfaktoren zu reifen dendritischen Zellen umbilden. Dazu muss der Patient einmalig an die Universitätshautklinik nach Erlangen reisen. Nach Beladung der Zellen mit der Tumor-RNA des Patienten (durch eine sog. Elektroporation) können diese aliquotiert eingefroren und nach Überprüfung der Qualitätskontrollen (Sterilität, Endotoxinfreiheit, Mykoplasmenfreiheit, Vitalität, Phänotyp, Zellzahl) an die zuweisenden Zentren zur Abgabe an den Patienten versendet werden. Die 


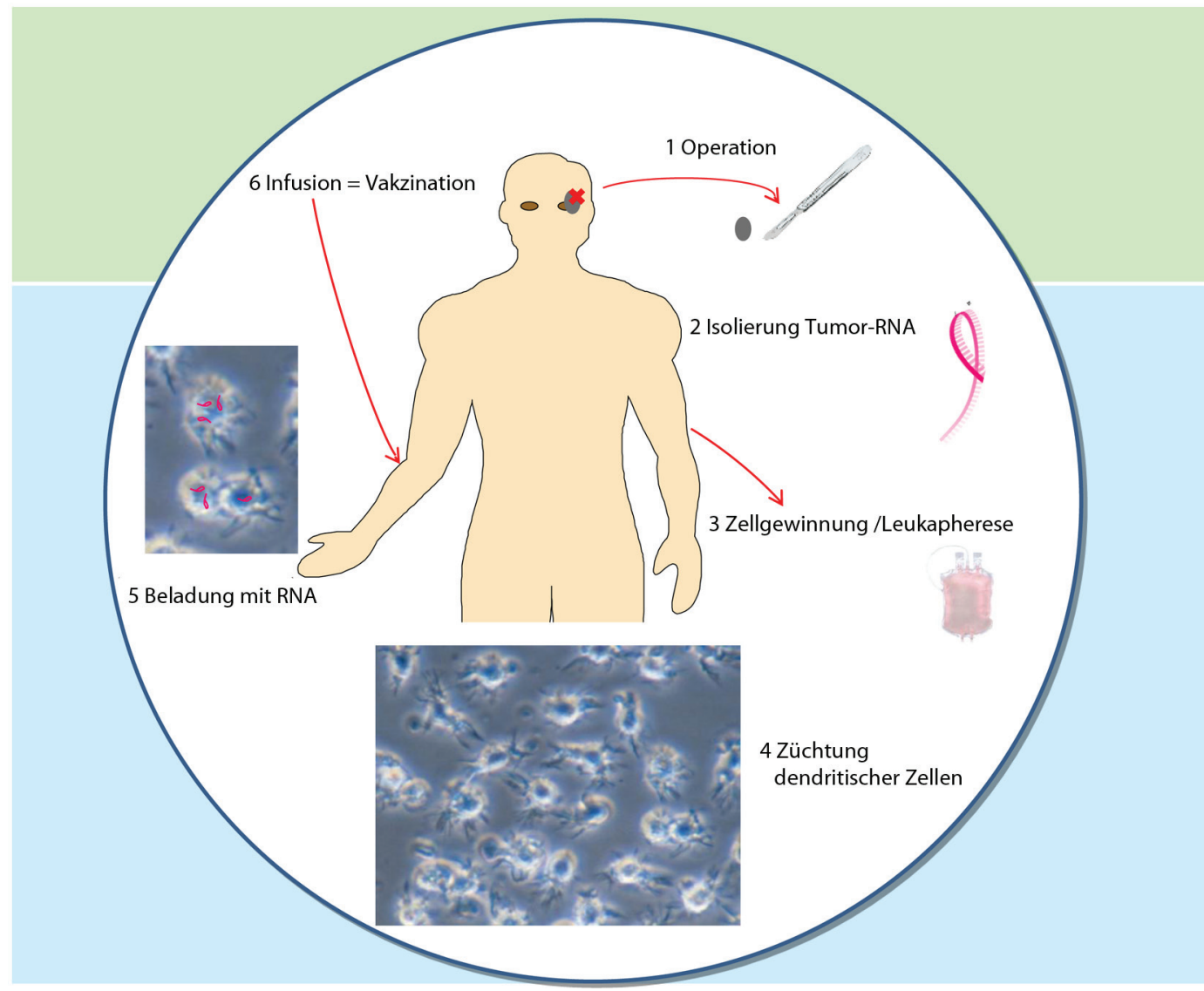

Abb. $1<$ Schematischer Ablauf der Tumorimpfung gegen uveale maligne Melanome

komplexe Prozedur der Tumor-RNAGewinnung aus geringen Tumorgewebemengen wurde als Kooperation der Augenklinik und Hautklinik des Universitätsklinikums Erlangen entwickelt.

Die Impfungen erfolgen am teilnehmenden Zentrum insgesamt 8-mal über einen Zeitraum von 2 Jahren als intravenöse Infusionen mit ansteigenden Intervallen (2, 4, 6 Wochen, 3 Monate, dann halbjährliche Intervalle). Durch die Vakzination kommt es zur Aktivierung zytotoxischer T-Zellen, die gegen jene Tumorantigene gerichtet sind, die nun durch die ex vivo beladenen dendritischen Zellen präsentiert werden, und die ein Abbild des Tumorantigenrepertoirs des jeweiligen Uveamelanoms darstellen.

Die Lebensqualität ist für die behandelten Patienten nahezu unbeeinträchtigt. Nebenwirkungen bestehen in nach den Impfungen auftretenden, kurz dauernden fieberhaften Episoden mit grippalen Symptomen, sehr selten Exanthemen oder Auftreten von Vitiligo.

\section{Fazit für die Praxis}

- Diese innovative Phase-III-Studie eröffnet erstmals Perspektiven auf ein längerfristiges Überleben auch für Patienten mit Hochrisikomelanom der Aderhaut. Alle potenziell inkludierbaren Patienten sollten von der Möglichkeit der Studienteilnahme erfahren und bei Wunsch daran teilnehmen können.

- Teilnehmen können Patienten mit großen, unbehandelten Uveamelanomen (T2-T4, AJCC TNM grading 2009) im Alter bis zu 75 Jahren.

- Ausschlusskriterien sind aktive Infektionen mit z. B. HIV oder Hepatitis, Zustand nach Organtransplantation und Zweitmalignom in den letzten 5 Jahren (Ausnahme nichtmelanozytärer Hautkrebs und Zervixkarzinom).

- Genauere Informationen über die Studie sind online unter https:// clinicaltrials.gov/ct2/show/ NCT01983748 nachzulesen.

\section{Korrespondenzadresse}

PD Dr. univ. B. Schuler-Thurner Experimentelle Immuntherapie, Hautklinik Universitätsklinikum Erlangen Hartmannstr. 14, 90152 Erlangen beatrice.schuler-thurner@uk-erlangen.de

\section{Einhaltung ethischer Richtlinien}

Interessenkonflikt. K.-U. Bartz-Schmidt, N. Bornfeld, C. Cursiefen, B. Fuisting, S. Grisanti, L.M. Heindl, L. Holbach, M. Keserü, H. Knorr, K. Koch, F. Kruse, R. Meiller, C. Metz, T. Meyer-terVehn, M. Much7, M. Reinsberg, S. Schliep, B. Seitz, G. Schuler, B. Schuler-Thurner, D. Süsskind, A. Viestenz, L. Wagenfeld und M. Zeschnigk geben an, dass kein Interessenkonflikt besteht.

Alle im vorliegenden Manuskript beschriebenen Untersuchungen am Menschen wurden mit Zustimmung der zuständigen Ethikkommission, im Einklang mit nationalem Recht sowie gemäß der Deklaration von Helsinki von 1975 (in der aktuellen, überarbeiteten Fassung) durchgeführt. Von allen beteiligten Patienten liegt eine Einverständniserklärung vor.

Open Access Dieser Artikel unterliegt den Bedingungen der Creative Commons Attribution License. Dadurch sind die Nutzung, Verteilung und Reproduktion erlaubt, sofern der/die Originalautor/ en und die Quelle angegeben sind. 


\section{Literatur}

1. Singh AD, Topham A (2003) Incidence of uveal melanoma in the United States: 1973-1997. Ophthalmology 110(5):956-961

2. Metz CH, Lohmann D, Zeschnigk M, Bornfeld N (2013) Uveal melanoma: current insights into clinical relevance of genetic testing [in German]. Klin Monbl Augenheilkd 230:686-691

3. Lipski AC, Lakotka N, Riechardt Al, Willerding GD, Heufelder J, Türkmen S, Keilholz U, Moser L, Joussen AM (2013) Diagnosis of and therapy for choroidal melanoma [in German]. Klin Monbl Augenheilkd 230:1005-1019

4. Heindl LM, Hofmann TN, Schrödl F, Holbach LM, Kruse FE, Cursiefen C (2010) Intraocular lymphatics in ciliary body melanomas with extraocular extension: functional for lymphatic spread? Arch Ophthalmol 128:1001-1008

5. Schroedl F, Brehmer A, Neuhuber WL, Kruse FE, May CA, Cursiefen C (2008) The normal human choroid is endowed with a significant number of lymphatic vessel endothelial hyaluronate receptor 1 (LYVE-1)-positive macrophages. Invest Ophthalmol Vis Sci 49:5222-5229
6. Schlereth SL, Neuser B, Herwig MC, Müller AM, Koch KR, Reitsamer HA, Schrödl F, Cursiefen C, Heindl LM (2014) Absence of lymphatic vessels in the developing human sclera. Exp Eye Res 125:203-209

7. Prescher G, Bornfeld N, Hirche H, Horsthemke B, Jöckel KH, Becher R (1996) Prognostic implications of monosomy 3 in uveal melanoma. Lancet 347(9010):1222-1225

8. Tarlan B, Kiratli H (2012) Current Treatment of choroidal melanoma. Expert Rev Ophtalmology 7(2):189-195

9. Shields J A, Shields C L (2015) Management of Posterior Uveal Melanoma: past, present, and future: the 2014 Charles L. Schepens Lecture. Ophthalmology 122(2):414-428

10. Moser JC, Pulido JS, Dronca RS, McWilliams RR, Markovic SN, Mansfield AS (2015) The Mayo Clinic experience with the use of kinase inhibitors, ipilimumab, bevacizumab, and local therapies in the treatment of metastatic uveal melanoma. Melanoma Res 25(1):59-63
11. Schuler G, Schuler-Thurner B, Steinman RM (2003) The use of dendritic cells in cancer immunotherapy. Curr Opin Immunol 15(2):13847. (Review)

12. Wilgenhof $S$, Corthals J, Van Nuffel AM, Benteyn D, Heirman C, Bonehill A, Thielemans K, Neyns B (2015) Long-term clinical outcome of melanoma patients treated with messenger RNAelectroporated dendritic cell therapy following complete resection of metastases. Cancer Immunol Immunother 64(3):381-388

\title{
Hier steht eine Anzeige.
}

\author{
블 Springer
}

